\title{
Mit vereinten Kräften: Letzte Chance einer Stabilisierung des neuen Südafrikas
}

\author{
Klaus Freiherr von der Ropp
}

Eingegangen: 13. Juli 2021 / Angenommen: 28. August 2021 / Online publiziert: 13. September 2021 (C) Springer Fachmedien Wiesbaden GmbH, ein Teil von Springer Nature 2021

Zusammenfassung Mit dem Ende des Kalten Krieges sahen sich Großbritannien und die USA imstande, Südafrika zur Aufgabe seiner in etwa 350 Jahren gewachsenen Politik der Apartheid zu zwingen. Der folgende Weg zur Demokratie war mit zahlreichen Defiziten behaftet. Dazu zählt der ehrgeizige Versuch der Bundesrepublik, Südafrika das deutsche Verfassungsrechtsmodell ,überzustülpen“. Das Modell erwies sich als brüchig und führte die junge Demokratie mit in den Abgrund. Der Beitrag positioniert sich zugunsten einer stärkeren politischen Einbindung der afrikaansen Bürgerrechtsbewegung Solidariteit, um den entstandenen Schaden zumindest zu begrenzen.

Schlüsselwörter Südafrika · Deutsches Verfassungsrechtsmodell ·

Minderheitenschutz · Implosion · Solidariteit · Afrikaaner-Afrika Initiative ·

Restabilisierung

Der Autor war von 1975 bis 1999 Leiter des Bonner Verbindungsbüros der Stiftung Wissenschaft und Politik und verfügt über eine jahrzehntelange Erfahrung als freiberuflicher Konsultant für Fragen des südlichen Afrikas.

Dr. Klaus Freiherr von der Ropp $(\square)$

Potsdam, Deutschland

E-Mail: Klaus@africanquestions.org 


\title{
With Joined Forces: Last Chance of Stabilizing the New South Africa
}

\begin{abstract}
With the end of the Cold War, Great Britain and the United States saw themselves in a position to force South Africa to abandon its policy of apartheid, which had grown over the past 350 years. The ensuing path to democracy was fraught with numerous shortcomings. These include Germany's ambitious attempt to "impose" the German constitutional model on South Africa. The model proved fragile and led the young democracy into the abyss. The article positions itself in favour of a stronger political involvement of the Afrikaner civil rights movement Solidariteit to limit the damage done.
\end{abstract}

Keywords South Africa - German constitutional model · Protection of minorities · Implosion $\cdot$ Solidariteit $\cdot$ Afrikaner-Africa Initiative $\cdot$ Restabilisation

\section{Einleitung}

Anfang 2020 kam Covid-19 wie ein Orkan über Südafrika. Die Pandemie traf dabei auf ein Land, das nach Jahrzehnten nicht selten blutiger Auseinandersetzungen im Inneren sowie einem Vierteljahrhundert einer sehr anspruchsvollen Übergangsphase bereits am Boden lag (Pabst und Ropp 2011). Schon zur Zeit des historischen Umbruchs im Mai 1994 befand sich die Wirtschaft der Kap-Republik in einer desolaten Verfassung (Roche 1998, S. 10). Denn nach Phasen starken Wachstums zeigten die mit Beginn der 1960er-Jahre verhängten und seither immer härter gewordenen Sanktionen der Außenwelt sowie der wachsende Widerstand gegen Apartheid im Inneren Wirkung. So verzeichnete Südafrika in der Dekade vor der ersten demokratischen Wahl (April 1994) in vier von zehn Jahren ein negatives Wirtschaftswachstum (The World Bank 2021a). Gleichzeitig ist die Bevölkerungszahl rasant gestiegen: von 31,8 Mio. Menschen im Jahr 1984 auf 40,6 Mio. im Jahr 1994 und 59,3 Mio. im Jahr 2020 (The World Bank 2021b). Mit Ausbruch der Pandemie im Jahr 2020 stieg, um hier nur einige wenige der vielfach desaströsen Wirtschaftsdaten anzuführen, die ohnehin hohe Arbeitslosigkeit im engeren Sinn bis zum Ende des ersten Quartals des Jahres 2021 auf 32,6\% und die Arbeitslosigkeit im weiteren Sinn ${ }^{1}$ auf 43,2\% (Statistics South Africa 2021, S. 15). In der Gruppe der 15-24-jährigen lag die Arbeitslosigkeit im engeren Sinn im ersten Quartal 2021 bei 63,3\% (Statistics South Africa 2021, S. 30). Und sehr viele Arbeitslose beziehen, wenn überhaupt, nur minimale Sozialleistungen. Umso größer sind die Einnahmen und Vermögen der „Fat Cats“, der neuen Machthaber². Alles Vorstehende ist vor dem Hintergrund eines negativen Wirtschaftswachstums von 7\% des BIP im Jahr 2020 (The World Bank 2021a) zu sehen. Covid-19 trifft Südafrika umso härter, da viele der Infizier-

\footnotetext{
${ }^{1}$ Zur Unterscheidung der Definitionen von Arbeitslosigkeit im engeren und im weiteren Sinn siehe Statistics South Africa (2021, S. 12).

${ }^{2}$ Im vorliegenden Artikel wird, abweichend vom ZfAS-Standard, bei personenbezogenen Substantiven die männliche grammatikalische Form verwendet. Der Autor schließt damit Personen jeden Geschlechts gleichermaßen ein.
} 
ten häufig unter sehr schweren Vorerkrankungen, vor allem Tbc und/oder HIV/Aids, leiden.

Der vorliegende Beitrag zeigt Entwicklungen auf, die zu der aktuellen Lage Südafrikas beigetragen haben, die in Kap. 4 genauer beleuchtet wird. Die Darstellung der Entwicklungen setzt in der Zeit des Kalten Krieges an (Kap. 2). Denn nur so ist die besondere Rolle Deutschlands (Kap. 3) zu verstehen, das auf Betreiben Londons aus der gemeinsamen westlichen Diplomatie im Süden Afrikas mangels Sachkunde ausgeschlossen worden war. Umso engagierter war die Bundesrepublik in den 1990erJahren in dem Bestreben, durch die Entsendung hunderter von Beratern den Neuaufbau der Kap-Republik zu unterstützen. Diese hatten allerdings, wenn überhaupt, nur oberflächliche Landeskenntnisse, sodass das deutsche Engagement letztlich mit zur drohenden Implosion des neuen Südafrikas beitrug. Abschließend wird ein Lösungsansatz präsentiert, in dessen Mittelpunkt die afrikaanse Bürgerrechtsbewegung Solidariteit steht (Kap. 5 und 6). Ziel des Beitrags ist es, eine Diskussion über die prekäre Lage Südafrikas anzuregen, insbesondere da nicht nur in Deutschland viele Medien die Thematik nicht zu erfassen scheinen. ${ }^{3}$ Dabei positioniert sich der Autor zugunsten einer stärkeren politischen Einbindung von Solidariteit.

\section{Der mühselige Weg zur Demokratie}

Der Weg hin zum Umbruch des Vielvölkerstaates zur Demokratie war mühselig. Dazu trug entscheidend bei, dass die Kap-Republik mit ihren sehr großen Vorkommen an weltweit seltenen Rohstoffen und ihren strategisch bedeutsamen See- und Flughäfen ein wichtiger Schauplatz des Kalten Krieges war. So sprach kein Geringerer als der große Stratege Egon Bahr davon, der Südafrika-Konflikt berge ständig die Gefahr des dritten Weltkrieges in sich, sollte für ihn keine Verhandlungslösung gefunden werden (Geschke und Mack 1977, S. 8). Man bedenke, dass das Land seinerzeit über sechs fertiggestellte und eine siebte, noch im Bau befindliche Kernwaffe vom Typ Hiroshima verfügte. Entsprechend war die Zukunft Südafrikas Jahr für Jahr in den UN-Vollversammlungen Gegenstand heftigen Streits zwischen den Staaten des Globalen Südens und den ihnen verbündeten des sozialistischen Lagers einerseits und der Mehrzahl der westlichen Staaten andererseits. Einen seiner nicht wenigen Höhepunkte erlebte dieser Streit am 30. November 1973 als die UNVollversammlung auf Betreiben der damaligen Union der Sozialistischen Sowjetrepubliken (UdSSR) mit sehr großer Mehrheit die „Internationale Konvention zur Unterdrückung und Bestrafung des Verbrechens der Apartheid“ verabschiedete. Im Schatten dieses Konflikts stand jener um die Zukunft der früheren deutschen Kolonie Südwestafrika und des zunächst Völkerbunds- und später UN-Mandats Namibia. Südafrika hielt es bis 1990 in den Augen der Vereinten Nationen völkerrechtswidrig besetzt.

Auf Initiative der Organisation für Afrikanische Einheit (OAE) sowie der von den Vereinten Nationen als solche anerkannten Befreiungsbewegungen South West African People's Organisation (SWAPO), African National Congress (ANC) und Pan

\footnotetext{
${ }^{3}$ Eine Ausnahme stellt in Deutschland das Fachblatt Afrika Süd dar.
} 
Africanist Congress (PAC) wurde der Ton der UN-Debatten schnell sehr scharf. So forderte etwa der Vertreter Sambias die Vereinten Nationen auf, den (Weißen) Regierungen in Windhuk und Pretoria den Krieg zu erklären, um Namibia und Südafrika von ihnen zu befreien. Daher riefen Anfang 1977 der US-amerikanische UN-Botschafter Andrew Young, sein Stellvertreter Donald McHenry und der kurz zuvor in das Bonner Außenministerium eingezogene Hans Dietrich Genscher eine westliche „Kontaktgruppe“ ins Leben. Ihr gehörten die damaligen fünf westlichen Mitglieder des UN-Sicherheitsrates an (USA, Großbritannien, Frankreich, Bundesrepublik Deutschland und Kanada). Ihre Aufgabe war es, Südafrika zu veranlassen, Namibia freizugeben. In Washington und wohl mehr noch im Bonner Außenministerium wurde, unausgesprochen, der Plan verfolgt, die Kap-Republik nach der Freigabe Namibias dazu zu bringen, ihre in nahezu 350 Jahren gewachsene Politik der Rassentrennung (seit 1948 Apartheid genannt) schnellstmöglich zu Gunsten einer demokratischen Ordnung aufzugeben. Diese Politik der Regierung Schmidt/Genscher litt vom ersten Tag an darunter, dass der Außenminister und seine Mitarbeiter Namibia und Südafrika nicht aus eigener Anschauung kannten. Bewusst bezogen sie ihre Informationen kaum jemals aus Windhuk und Pretoria, sondern vornehmlich aus New York, dem Sitz der Vereinten Nationen. Gleichwohl machte Genscher diese Region von 1974 bis Ende der 1970er-Jahre zum Schwerpunkt seiner Außenpolitik. Denn nahezu alle anderen Felder westdeutscher Außenpolitik bestellte, im Rahmen seiner ihm nach dem Grundgesetz zustehenden Richtlinienkompetenz, Bundeskanzler Helmut Schmidt selbst (Steininger 2009, S. 7). Anders als Genscher in seinen ersten Jahren als Bundesminister des Auswärtigen war Schmidt ein weltweit anerkannter Außen- und Sicherheitspolitiker.

Die Diplomatie der Kontaktgruppe führte im September 1978 nach mühseligen Verhandlungen mit einem störrischen Partner (Südafrika) zur Verabschiedung von Resolution 435 des UN-Sicherheitsrates, dem völkerrechtlich verbindlichen Plan zur Entlassung Namibias in die staatliche Unabhängigkeit. Dessen Implementierung scheiterte aber abrupt im Oktober 1978. Vom 16. bis18. Oktober hielten sich auf Initiative Genschers er selbst und seine Kollegen aus Großbritannien (David Owen), den USA (Cyrus Vance) und Kanada (Donald Jamieson) sowie der französische Staatssekretär Olivier Stirn in Pretoria auf, um mit der südafrikanischen Regierung letzte Einzelheiten der Unabhängigkeit Namibias zu klären. Da die Mitglieder der Kontaktgruppe mit anhaltendem Widerstand Pretorias gegen die Freigabe Namibias rechneten, hatten sie im Vorfeld eine Liste härtester Wirtschaftssanktionen für den Fall anhaltender Unbotmäßigkeit Südafrikas erarbeitet. Diese Liste wurde allerdings auf Vorschlag der Briten, nicht auf Beschluss des UN-Sicherheitsrates erstellt. Dadurch fehlte es den angedachten Sanktionen an völkerrechtlicher Verbindlichkeit. Im Lauf der Verhandlungen richtete der südafrikanische Außenminister Roelof Botha am 17. Oktober 1978 an Genscher als dem Wortführer der Kontaktgruppe die Frage, ob sie diese Sanktionen für den Fall verhängen würden, dass Pretoria nicht voll kooperiere. Nachdem der Deutsche diese Frage bejaht hatte, fielen ihm Owen und, diesem folgend, Vance ins Wort und stellten fest, dass das für ihre Regierungen nicht gelte. Daraufhin lachte Botha seinen deutschen Kollegen schallend aus. Statt sich mit Genscher zu solidarisieren, schlossen London und Washington ihn - und damit die Bonner Regierung - für gut zehn Jahre aus allen internationalen 
Verhandlungen über die Zukunft Namibias und Südafrikas aus. Zu ergänzen bleibt, dass die Vertreter Kanadas und Frankreichs die Demütigung Genschers teilnahmslos hinnahmen.

Es wird sich wohl niemals klären lassen, ob es sich um ein von London und Washington einerseits und Pretoria andererseits abgekartetes Spiel handelte. Dies erscheint allerdings sehr wahrscheinlich, da der seinerzeit überforderte Bonner Außenminister ihnen in seinen ambitionierten diplomatischen Bemühungen um einen raschen Umbruch Südafrikas lästig war. Zu groß war die Sorge, dass eine neue Ausrichtung Südafrikas zu einer Zuwendung zur Sowjetunion führen könnte. In einem solchen Fall hätte erstens die Gefahr bestanden, dass sich ein Rohstoffkartell um Südafrika, Simbabwe und eben die UdSSR bildet, das für die Rüstungsindustrie wichtige Rohstoffe, insbesondere zur Stahlveredelung, exklusiv an Moskau liefert. Zweitens fürchteten vor allem Großbritannien und die USA, dass Südafrika in einem solchen Szenario zu einer unkontrollierbaren Quelle der Verbreitung von Atomwaffen sowie von Kenntnissen über die Technologie ihrer Herstellung werden könnte. Drittens hätte sich das wirtschaftlich besonders eng mit Südafrika verflochtene Großbritannien im Fall schwerer Unruhen im Zuge des Umbruchs mit der Evakuierung von mindestens 750.000 britischen Passinhabern konfrontiert gesehen (Pabst 2019, S. 25, 30; Ropp 2019, S. 59).

Dafür, dass London und Washington bemüht waren, die Position Genschers in den Verhandlungen zu schwächen, spricht auch, dass die westlichen Delegationsführer ohne Berater und/oder Protokollanten in die Verhandlung mit den Südafrikanern gegangen waren, wie Genschers Chefberater Hans-Joachim Vergau (2002) später berichtete.

Erst mit der Wahl von Michail S. Gorbatschow zum Generalsekretär der KPdSU im Jahr 1985 und dem damit nahenden Ende des Kalten Krieges sahen sich London und Washington in der Lage, ihre Namibia- und Südafrikapolitik wieder aufzunehmen. Die Bundesrepublik wurde weiterhin nicht mit eingebunden. Frankreich und Kanada blieben der Namibia- und Südafrikapolitik aus freien Stücken fern. Eine wertvolle Hilfe für US-Botschafter Princeton Nathan Lyman und dessen britische Kollegen Sir Anthony Reeve, seit 1991 Nachfolger des legendären Sir Robin - „His Excellent Exellency“ (Robertson 1991, S. 14) - Renwick, war die Unterstützung ihrer Diplomatie durch den gleichfalls herausragenden russischen Botschafter Jewgeni Petrovich Gusarow. Seine Aufgabe war es, mäßigend auf die Unterhändler der Allianz aus ANC, South African Communist Party (SACP) und Congress of South African Trade Unions (COSATU) einzuwirken. Dieser Allianz war die UdSSR in Zeiten des Kampfes gegen Apartheid über Jahrzehnte eng verbunden gewesen.

Die hochklassige britisch-amerikanisch-sowjetische/russische Diplomatie wurde schnell von Erfolg gekrönt: Gemäß Resolution 435 des UN-Sicherheitsrates wurde Namibia im März 1990 unabhängig. Ende 1991 nahm die südafrikanische Regierung, begleitet von der Androhung härtester Wirtschaftssanktionen durch die USA, mit den im Jahr zuvor entbannten Befreiungsbewegungen, zuvörderst der sehr gefolgschaftsstarken ANC/SACP/COSATU-Allianz, Verfassungsverhandlungen auf. Die Verhandlungen führten nach etwa zwei Jahren zur Verabschiedung einer im In- und Ausland hochgelobten demokratischen Verfassung. 
Zu der Handvoll von Beobachtern, die erkannten, dass die südafrikanische Verfassung mangels einer demokratischen und rechtsstaatlichen Kultur in der KapRepublik von Geburt an den Keim des Scheiterns in sich tragen würde, gehörte in Deutschland Egon Bahr. Angesichts der tiefen kulturellen Zerrissenheit des Landes hatte er bereits 1977 ein System des One man, one vote als für Südafrika untauglich bezeichnet. Stattdessen forderte er für Südafrika „ein bisher unbekanntes Modell des gleichberechtigten Zusammenlebens mit besonderem Schutz für Minderheiten“ (Geschke und Mack 1977, S. 8). Seine Warnung wurde jedoch überhört, ebenso wie die von Francis Fukuyama (1991), der die Sorge aussprach, Südafrika werde sich in der Zukunft nicht von Vorstellungen lösen können, die die neuen Regierenden von ihren Lehrmeistern im Exil in der DDR, in Kuba und in der UdSSR mit an die Südspitze des afrikanischen Kontinents gebracht hatten. Bahrs Warnung vor einem Scheitern des sehr ehrgeizigen südafrikanischen Transformationsprozesses wurde in Deutschland einzig von dem späteren Präsidenten der Liberalen Internationalen, Otto Graf Lambsdorff, ernstgenommen und weiterentwickelt. Lambsdorff (1986) redete der Schaffung eines „Afrikaaner Israel“, wahrscheinlich im westlichen Kap gelegen, das Wort. Dort sollten die Weißen (und sogenannten Coloured) Afrikaaner eine Fluchtburg (toevlugsoord) finden, die im übrigen, Schwarz dominierten Südafrika keine Zukunft für sich und ihre Kinder sähen. ${ }^{4}$

Die geäußerten Bedenken fanden jedoch keinen Eingang in die „Weltmeinung“, die in Deutschland inzwischen bei weitem nicht mehr nur von Genscher und seinen Parteigängern vertreten wurde und die einst in Teilen hochentwickelte Kap-Republik ins Verderben stürzen sollte. Künftige Historiker werden die Frage aufwerfen, weshalb das neue Südafrika diesen Weg in eine bloße Fassadendemokratie eingeschlagen hat. Sie werden darauf wohl zwei Antworten geben: zum einen das Versagen des auf der ganzen Linie in den Verhandlungen überforderten Staatspräsidenten Frederik Willem de Klerk und zum anderen die Beratung der Verfassungsgeber durch eine unüberschaubar große Zahl deutscher Fachleute, die allesamt Vertreter der „Weltmeinung" zur Neuordnung Südafrikas waren.

\section{3 ,German Verfassungsrecht under the Southern Cross“}

Das kurz zuvor wiedervereinigte Deutschland war mehr als jeder andere Drittstaat bereit, bei dem verfassungsrechtlich-politischen Aufbau des neuen Südafrika mitzuwirken (Ropp 2013), nicht zuletzt vor dem Hintergrund des demütigenden Ausschlusses aus allen Verhandlungen über Südafrika und Namibia seit Oktober 1978.

Mit großem Aufwand wurden hunderte von Beratern an das Kap entsandt. Sie kamen u. a. aus dem Bundestag und etlichen Landtagen, aus dem Bundesrat, aus Bundes- und Landesministerien, aus einer Vielzahl von Kommunen, von allen sechs politischen Stiftungen, aus vielen Universitäten, vom Bundesverfassungsgericht, vom

\footnotetext{
${ }^{4}$ Begriffe wie Coloured, Schwarz, oder Weiß werden im Sinne einer sozialen Klassifizierung verwendet, wie es unter der Apartheid der Fall war. Der Autor distanziert sich von dieser Kategorisierung aus Zeiten der Apartheid, verwendet die Begriffe aber aus Gründen der Verständlichkeit sowie aus der Notwendigkeit heraus, sich auf in dieser Weise definierte Statistiken zu beziehen.
} 
Bundesrechnungshof, von der damaligen Deutschen Gesellschaft für Technische Zusammenarbeit, von der Bundeswehr, vom Deutschen Gewerkschaftsbund oder von der Evangelischen Kirche in Deutschland. Die Bundesrepublik sah sich dazu befähigt, Südafrika umfassend bei dem verfassungsrechtlich-politischen Aufbau zu beraten, weil sie infolge der Jahrhundertkatastrophe des Dritten Reichs seit 1949 bzw. 1990 eine zu Recht weltweit als vorbildlich anerkannte Verfassung hat (Grimm 2020). Diese wurde dem Post-Apartheid-Südafrika von in- und ausländischen Beratern schlichtweg, ,übergestülpt“ - mit Ausnahme ihrer föderalen Strukturen, die die ANC/SACP/COSATU-Allianz mit guten Gründen nicht bereit war zu übernehmen. Nicht erst heute stellt sich die Frage, ob sich die deutsche Verfassung so nahtlos wie seinerzeit gedacht auf einen völlig anderen Kulturkreis übertragen lässt. Zumindest den aus Deutschland angereisten Beratern hätte klar sein müssen, dass das deutsche Grundgesetz zunächst im Westen und dann auch im Osten nur unter sehr besonderen Umständen zustande gekommen war.

In der Literatur wird das eifrige deutsche Engagement unterschiedlich bewertet. So kommt etwa der südafrikanische Verfassungsrechtler Lourens Marthinus du Plessis (2008) in seinem Aufsatz „German Verfassungsrecht under the Southern Cross“" zu dem Schluss, dass das deutsche Verfassungsrecht eine wertvolle Quelle für das Zustandekommen der südafrikanischen Verfassung war. Dagegen stellte die hochqualifizierte US-Journalistin Patti Waldmeir (1991, S. 3) kurz und bündig fest: „Democratic niceties will have to wait upon the overwhelming need to restore stability“. Denn auch für Südafrika gilt, was der Heidelberger Politikwissenschaftler Klaus von Beyme (2001) zum postsowjetischen Russland feststellte: „Demokratie ist nicht gegeben, Demokratie ist aufgegeben“.

Unabhängig von der generellen Frage der Übertragbarkeit von Verfassungssystemen enthielt der 1993/94 in Kempton Park Johannesburg verabschiedete Verfassungsentwurf eine für das kulturell tief zerrissene Südafrika fatale Unterlassung: Er verzichtete auf jeden substanziellen Minderheitenschutz. Daraufhin riefen die Botschafter Lyman und Reeve, denen die Warnungen Bahrs und Lambsdorffs vor der Instabilität des neuen Südafrika in allen Einzelheiten vorlagen, die Spitzen des ANC um Thabo Mbeki $^{5}$ und der Freedom Front ${ }^{6}$ ab August 1993 zu Beratungen zusammen. Das Ziel war die Aushandlung des, so sein späterer offizieller Titel, ,Accord on Afrikaner Self-Determination between the Freedom Front, the African National Congress and the South African Government/National Party“. Der Accord wurde von den weltweit anerkannten afrikaansen Dissidenten Frederik van Zyl Slabbert (Grundlingh 2021) und Breyten Breytenbach unter Hinzuziehung des Verfassers des vorliegenden Beitrags formuliert. Als historisches Vorbild diente die verfassungsrechtliche Stellung der Russlanddeutschen in Russland/Sowjetunion. Am 23. April 1994 wurde der Accord im Union Building, dem Sitz der südafrikanischen Regie-

\footnotetext{
5 Thabo Mbeki war über viele Jahre führender Aktivist des ANC und bekleidete von 1999 bis 2008 das Amt des südafrikanischen Staatspräsidenten.

${ }^{6}$ Bei der Freedom Front handelte es sich um eine Neugründung konservativer, aber nicht reaktionärer, Weißer Afrikaaner um den landesweit anerkannten früheren Viersternegeneral Constand Viljoen. Das starke Lager der Weißen Reaktionäre versuchte dagegen, zur Apartheid zurückzukehren. Das führte zu einem radikalen Bruch mit Viljoen.
} 
rung, in Anwesenheit von u. a. Lyman, Reeve und dem Verfasser des vorliegenden Beitrags (als Beobachter der EU) von Mbeki, Viljoen und dem Verfassungsminister der Regierung de Klerk, Roelf Meyer, unterzeichnet (The Nelson Mandela Foundation o.J.; Cruywagen 2014). Zweierlei war hier auffällig: Der deutsche Botschafter Hans-Christian Ueberschaer und sein Stab blieben, obwohl eingeladen, der Unterzeichnung demonstrativ fern. Und der russische Botschafter Gusarow begrüßte die Unterzeichnung wenige Tage später bei einem Treffen mit Viljoen überschwänglich. Obwohl er in verwässerter Form (Artikel 235 und 185) in die Verfassung aufgenommen wurde, wurde der Accord, der den Afrikaanern innere Autonomie zugesteht, bislang nicht in die Praxis umgesetzt. Das wurde durch die tiefe Zerstrittenheit der zahlreichen Parteigänger Viljoens verhindert.

\section{Die Gefahr der Implosion}

Die 1994 in allen Bevölkerungsgruppen durchaus vorhandene Zuversicht auf das Gelingen des demokratischen Neubeginns gründete sich vor allem auf die Einzigartigkeit des ersten gewählten südafrikanischen Staatspräsidenten Nelson R. Mandela (1994-1999). Er allein verkörperte durch seine übermenschliche Bereitschaft zur Versöhnung den Glauben an den Wiederaufstieg des darniederliegenden Landes. Von ihm wurde allgemein erwartet, dass seine Politik das bekannte Schlagwort des ANC, , a better life for all“, in die Tat umsetzen werde, nicht zuletzt auch im wirtschaftlichen Bereich. Ein Vierteljahrhundert später ist wenig von seinen Bemühungen übriggeblieben. So stellt Robert Kappel (2019), ein ausgesprochener Kenner der südafrikanischen Volkswirtschaft, zu Recht fest, ,dass tiefe Strukturprobleme, die zu ineffizienter Ressourcenallokation beitragen und nachteilige Wirkung auf die Beschäftigung haben, sich seit dem Machtwechsel in Pretoria nicht verringert, sondern sogar vergrößert haben“. Bezeichnend sollte sein, dass Anfang 2020 mit Moody’s die dritte und letzte der großen Ratingagenturen die Kreditwürdigkeit Südafrikas auf Ramschniveau herabgestuft hat.

Die wichtigste Ursache für den drastischen Niedergang Südafrikas (Johnson 2021a; 2021b) ist, dass das Land seit seiner Hinwendung zur Demokratie nicht mehr über eine effiziente staatliche Verwaltung verfügt. Sie war bis zur Machtumkehr zweifelsfrei die wichtigste Stütze des Ancien Régime und wurde daher faktisch abgeschafft. Was folgte, war oft nicht mehr als nacktes Chaos.

Aus allen seit 1994 offenbar weitgehend korrekt ${ }^{7}$ durchgeführten Parlamentswahlen ist die bis heute unangefochten alleinregierende ANC/SACP/COSATU-Allianz als deutlicher Sieger hervorgegangen. Entsprechend groß ist ihre Machtfülle, und so konnte sie von Anfang an ihre Politik der Affirmative Action verfolgen. Diese ,is aimed at ensuring that black South Africans, women, and other disadvantaged groups who had been marginalized and unfairly discriminated against in the past, are provided with access to opportunities“ (Makoba und Ntebeng 2002, S. 165). Die

\footnotetext{
7 Eine Besonderheit gilt allerdings für die erste Wahl. Im Interesse der schwächeren Parteien wurde nämlich ihr Ergebnis nicht an den Urnen ermittelt, sondern vorab ,ausgehandelt“ (Waldmeir und Holman 1994).
} 
südafrikanische Politik der Affirmative Action und insbesondere deren Umsetzung ist Gegenstand zahlreicher Betrachtungen, die zu unterschiedlichen Bewertungen kommen (Adam 1997; Makoba und Ntebeng 2002; Alexander 2007; Ratuva 2013; Erasmus 2015; Cuddihy 2016).

Affirmative Action führte u. a. zur Ersetzung einer sehr großen Zahl gut ausgebildeter, in der Regel Weißer, im westlichen Kap oft Coloured, Staatsdiener durch politisch genehme, jedoch oftmals wenig qualifizierter Schwarzer Beamter. Dieser Vorgang vollzog sich in Ministerien, bei der Polizei, bei Grenzsicherung/ Einwanderungskontrolle, im Justizwesen (inklusive Strafvollzug), in den Streitkräften, in den Kommunalverwaltungen, in den Universitäten, im Krankenhauswesen, bei der Trinkwasseraufbereitung und der Abwasserentsorgung. Mit Blick auf die Effizienz des öffentlichen Sektors hält Erasmus (2015, S. 107) fest: „Affirmative action policy in itself is not the key barrier to efficiency in this sector. Instead, ,cadre deployment" as a key method of affirmative action in South Africa's public sector is the primary cause of mediocre service delivery".

Affirmative Action mit negativen Folgen gab es ferner bei vielen der ca. 300 staatseigenen Unternehmen (ESKOM, Trasnet, SAL, u. a.), weil viele der neuen Mitarbeiter ihren Aufgaben nicht gewachsen waren. Das alte Südafrika hatte sie im Rahmen seiner (rudimentären) Bantu Education nicht für diese Aufgaben ausgebildet. ${ }^{8}$ Auch hier hat das Post-Apartheid-Südafrika die Chance eines Neubeginns verpasst. So gilt nach wie vor, was die in der Zeit der Apartheid lange Jahre gebannte Bürgerrechtlerin Mamphela Ramphele (2012a) verschiedentlich feststellte: Das heutige Schulwesen ist (noch) schlechter als dasjenige, für dessen Überwindung viele Jugendliche bei den Aufständen von 1976 starben. Ihr ist zuzustimmen, wenn sie weiterhin feststellt: „South Africa is seen to be in the grip of self-sabotage“ (Ramphele 2012b). Hier muss der Hinweis genügen, dass das World Economic Forum (2017, S. 268) die Qualität des südafrikanischen Bildungswesens in der Kategorie Health and Primary Education auf Platz 121 von 137 untersuchten Ländern einstuft. Mit Blick auf die Qualität der mathematisch-naturwissenschaftlichen Ausbildung in der Kategorie Higher Education belegt Südafrika sogar nur Platz 128 von 137 (World Economic Forum 2017, S. 269).

Verschiedene Maßnahmen im Rahmen der Affirmative Action werden noch für sehr lange Zeit die Wurzel für den Absturz ganzer Regionen in die Anarchie sein. So sind etwa viele der sogenannten Townships wiederholt Ausgangspunkt gewaltsamer, krimineller Handlungen (Soest 2020). Hier bleibt den Opfern mangels öffentlicher Sicherheit und Ordnung oftmals bloß die Lynchjustiz. Im ländlichen Bereich ist das allerding da anders, wo an Stelle der seit 1994 gewählten Körperschaften jetzt wieder wie in Apartheidzeiten traditionelle Autoritäten (,traditional chiefs") das Sagen haben.

Landesweit verbreitet ist schließlich eine hemmungslose Korruption epidemischen Ausmaßes (Bond 2014). Viele der staatseigenen Unternehmen wurden von

\footnotetext{
8 „The Apartheid system created educational inequalities through overt racist policies. The Bantu Education Act of 1952 ensured that Blacks receive an education that would limit educational potential and remain in the working class" (Ocampo 2004). Einen umfassenden Überblick zu der Thematik und zu den bis heute spürbaren Auswirkungen bietet Hunter (2019).
} 
Mitarbeitern, die sie der Affirmative Action wegen (,Zuptagate“) einstellen mussten, geplündert (Swilling et al. 2017). Der dramatische Niedergang Südafrikas wird sich weiter beschleunigen, sollte es dem bereits heute im ANC sehr gefolgschaftsstarken Generalsekretär Elias („Ace“) Magashule gelingen, den heutigen ANC-Präsidenten und Staatspräsidenten Cyril Ramaphosa aus seinen Ämtern zu verdrängen (Poplak 2021). Denn Magashule, offenbar durch und durch korrupt, wird zusammen mit dem hochkriminellen früheren Staatspräsidenten (2009-2018) Jacob Zuma, dessen nicht minder krimineller, starker Klientel und Julius Malema, dem „Oberkommandierenden“ der Economic Freedom Fighters, einer 2014 entstandenen militanten Absplitterung des ANC, Südafrika wohl ,nach Simbabwe“, d. h. in die völlige Zerstörung führen. Vielleicht gibt Hoffnung, dass Magashule seit Mai 2021 von seinem Amt suspendiert ist. Getrübt wird diese Hoffnung indes durch die schweren, teils bürgerkriegsähnlichen Unruhen, von denen Südafrika vor allem in den Provinzen KwaZulu-Natal und Gauteng seit Juli 2021 heimgesucht wird (Tagesschau 2021; March 2021). Ausgelöst wurden sie durch die Inhaftierung des immer noch gefolgsstarken früheren Staatspräsidenten Jacob Zuma. Er hatte sich zuvor geweigert, vor einer Untersuchungskommission (Zondo Kommission) zur Aufklärung der ungezählten Korruptionsaffären während seiner Amtszeit auszusagen. Daraufhin war er vom Verfassungsgericht wegen Missachtung der Justiz zu einer Beugehaft von 15 Monaten verurteilt worden. Zunächst waren es mehr oder weniger ausschließlich seine Parteigänger, die nach seiner Inhaftierung wirtschaftlich wichtige Regionen mit Plünderung, Brandstiftung, Diebstahl, Raub und Mord überzogen. Binnen weniger Tage schlossen sich ihnen ungezählt viele Menschen an, wobei die Straßenkämpfe in den Stadtzentren von u. a. Johannesburg (inkl. Soweto), Durban und Pietermaritzburg, d. h. wirtschaftlichen Hochburgen, stattfanden.

Eine Orientierung in Richtung Magashule, Zuma und/oder Malema dürfte zu einer Umsetzung der aktuell noch strittig diskutierten Pläne führen, die Verfassung dahingehend zu ändern, dass sie künftig die entschädigungslose Enteignung privaten Eigentums (also nicht nur von Grund und Boden) zulässt. Bereits die bloße Möglichkeit einer solchen Gesetzesänderung hat in den zurückliegenden Jahren zu einem mangels zuverlässiger Statistiken nicht zu beziffernden Abfluss von aus- und inländischem Kapital geführt. Noch viel ernster ist die Abwanderung hunderttausender, in der Regel gut ausgebildeter Fachleute (Bröll 2008). Ihren Exodus würde die Kap-Republik nach Einschätzung des Autors in den nächsten weit mehr als 50 Jahren nicht verkraften. Genau dies könnte aber aufgrund der genannten politischen Entwicklungen in einem großen Ausmaß erfolgen. Dazu beitragen wird ferner die Bevölkerungsentwicklung: Aufgrund der Zuwanderung ${ }^{9}$ aus den bankrotten Nachbarstaaten ${ }^{10}$ sowie eines deutlichen Geburtenüberschusses sind heute ca. $81 \%$ der rund 59 Mio. Einwohner Südafrikas Schwarz. Nur noch rund 9\% gehören zur Gruppe der ganz überwiegend afrikaanssprachigen Coloureds; gut 2,5\% stammen aus Asien, insbesondere der früheren britischen Kolonie Indien. Die Weiße Bevöl-

\footnotetext{
9 Einen differenzierten Überblick über diese Thematik bietet z. B. Runge (2015).

10 Mangels effizienter Einwanderungskontrolle wanderten seit 1994 Millionen Bürger anderer afrikanischer Staaten und Pakistans illegal nach Südafrika ein. Allein aus dem bankrotten Simbabwe waren es laut inoffiziellen Schätzungen über drei Millionen.
} 
kerungsgruppe umfasst noch knapp $8 \%$; ca. $61 \%$ von ihnen sind afrikaanssprachig, die übrigen vorwiegend englischsprachig (Government of South Africa 2021). ${ }^{11}$ Diese Entwicklung ist bei vielen Afrikaanern mit der Sorge einer Marginalisierung verbunden. Den drohenden Machtverlust vor Augen, mehrten sich bereits in den frühen 1990er-Jahren vor allem unter den Parteigängern Viljoens die Stimmen, die das Existenzrecht der afrikaanssprachigen und anderer ethnischer Minderheiten durch die Ausgliederung eines „Volksstaates“ (Afrikaaner-Israel) aus Südafrika sicherzustellen bemüht waren. Sie griffen damit auf Überlegungen des konservativen, oftmals kritisch betrachteten (Ropp 2018; Weber 2011; Webster 2019), aber nach Einschätzung des Autors nicht reaktionären Theologen Carel Boshoff zurück, der seit den späten 1970er-Jahren an solchen Plänen arbeitete (Ropp 2017). Boshoff wiederum hatte hier wissenschaftliche Beiträge zu der Thematik vor Augen, die außer in Südafrika vor allem in Deutschland publiziert worden waren (Zollmann 2021). Zumindest derzeit haben aber wohl noch die Worte des Politikwissenschaftlers Deon Geldenhuys (2021, S. 100) Gültigkeit: ,Solidariteit regards as neither achievable nor workable under present conditions [...] an independent volksstaat for Afrikaners“. Der regierenden Allianz sollte allerdings klar sein, wovor der einst mächtige $\mathrm{Zu}$ luführer Mangosuthu Buthelezi in Apartheidzeiten seine vielen Gegner mit Recht warnte: „Better than most I know the preparedness of South Africa's Whites to scorch the earth and to die in defense of the indefensible". Entsprechend stellte das liberale südafrikanische Institute of Race Relations Anfang 2020 in einem Flugblatt fest: „South Africa is again at the brink“. In der Frage, ob das Land die Schwelle in den Abgrund überschreitet, könnte der afrikaansen Bewegung Solidariteit eine entscheidende Rolle zukommen.

\section{Die Rolle von Solidariteit}

Die Afrikaaner könnten auch heute noch ein wichtiger Faktor in der Stabilisierung des neuen Südafrikas sein. Denn sie verfügen über die Erfahrung und das Potenzial, durch Mitarbeit in der staatlichen Verwaltung und in den staatseigenen Wirtschaftsunternehmen das Land zu stabilisieren. Sollte die ANC/SACP/COSATU-Allianz jemals bereit sein, der Empfehlung des chinesischen Botschafters in Pretoria, Liu Songtian, zu folgen und sich mit dem Internationalen Währungsfonds (IWF) ins Benehmen zu setzen (Winning und Bavier 2019), sollte eine der ersten Maßnahmen dieser Kooperation sein, da wo Bedarf besteht, auf das Humankapital der nach 1994 angehalfterten Staatsdiener (inklusive der Mitarbeiter bei den staatseigenen Unternehmen) zurückzugreifen.

So verfügt von allen ethnischen Minderheiten vor allem die der rund 4,7 Millionen Weißen Südafrikaner im neuen Südafrika noch über Ressourcen, die zur Überwindung verschiedener Probleme im Land beitragen könnten. Und sie weiß sie zu nutzen. Am deutlichsten wurde das 2001, als der sehr überzeugende Gewerkschaftsführer Flip Buys nach dem Vorbild der polnischen, bewusst christlichen Bürger-

${ }_{11}$ Zum Vergleich: Im Jahr 1996 ordneten sich 76,7\% der damals 40,6 Mio. Einwohner Südafrikas als Afrikaner, 10,9\% als Weiße, 8,9\% als Coloureds und 2,6\% als Inder/Asiaten ein (GCIS 2001, S. 1). 
rechtsbewegung Solidarnosc deren südafrikanisches Pendant, Solidariteit, gründete. Sie erhält keine staatlichen Zuwendungen, finanziert sich mithin ausschließlich aus Beiträgen und Spenden. Außer an Solidarnosc orientiert sich Solidariteit mit ihren ca. 20 Institutionen an der Arbeit der israelischen Histradut. Ohne die Existenz von Solidariteit wäre der Aderlass an Fachkräften, der längst bedrohliche Ausmaße angenommen hat, noch viel größer. Denn immer mehr von ihnen würden es Anne Paton, der Witwe des Weißen Rebellen und Autors des weltberühmten Anti-Apartheid Romans „Cry the beloved country“ Alan Paton gleichtun: Bereits vor einem Vierteljahrhundert verließ sie Südafrika mit den Worten: „Fly the beloved country“.

Unter den Institutionen von Solidariteit sind die Gewerkschaft gleichen Namens mit immerhin 200.000 Mitgliedern sowie die Organisation Helpende Hand mit ihren Sozialprogrammen für die seit der Machtumkehr drastisch gestiegene Zahl armer Afrikaaner am bekanntesten. Dazu kommt AfriForum, zu dessen Aufgaben (mit seinen ca. 130 Ortsvereinen) nicht zuletzt die Bekämpfung von Kriminalität zählt. Angesichts der Ineffizienz großer Teile der Polizeicorps ist AfriForum Garant für die Aufrechterhaltung von Law and Order. Große Erfolge erzielen auch Sol-Tech, eine Fachhochschule für Ingenieurwesen mit 1600, und eine Universität, „Akademia“, mit 1000 Studenten, die hier in ihrer Muttersprache Afrikaans unterrichtet werden. Daneben unterhält Solidariteit ein großes, für seine Leistungen landesweit anerkanntes Forschungsinstitut und afrikaanssprachige Medien (Maroela Media und Radio Pretoria FM). Nach eigenen Angaben hat Solidariteit heute 330.000 Mitglieder und vertritt angeblich die Interessen von 500.000 Familien. Große Bedeutung hat ferner die Führung gerichtlicher Musterprozesse.

Solidariteit erbringt für ihre afrikaansen Mitglieder und teilweise auch weitere Bürger Dienstleistungen, die der daniederliegende Staat nicht mehr erbringen kann. Solidariteit begegnet damit der Furcht vor einem Übergreifen der von der regierenden Allianz zu verantwortenden anarchischen Zustände in vielen Townships auf andere Siedlungen. Wichtig sind auch Nachbarschaftswachen zur (zumindest) Eindämmung der vielerorts grassierenden Kriminalität, zumal die durch Covid-19 stark angestiegene Arbeitslosigkeit immer mehr Menschen in Vermögensdelikte, nicht selten in Verbindung mit Tötungsdelikten, treibt. Weiter hat AfriForum es übernommen, die breite Öffentlichkeit etwa über Morde an Farmerfamilien und ihren Landarbeitern zu informieren. Daneben gibt es eine Fülle weiterer Aktivitäten: die Instandsetzung maroder Straßen und sonstiger Infrastruktur und ihre anschließende Instandhaltung, die Aufbereitung von Trinkwasser und die Entsorgung von Abwasser sowie die allgemeine Müllentsorgung. Ferner ist AfriForum dafür verantwortlich, Abhilfe für Probleme im Zusammenhang mit der Gewinnung von Schiefergas und beim Betrieb von Mautstraßen zu leisten (Geldenhuys 2021).

Seit kurzem unterhält Solidariteit schließlich eine private (!) Staatsanwaltschaft, die angesichts verbreiteter Passivität der National Prosecuting Agency Korruptionsdelikte, die andernfalls nicht geahndet werden, anklagt. Darüber hinaus engagiert sie sich gegen die weitere Verdrängung der afrikaansen Kultur aus der Öffentlichkeit, z. B. von Afrikaans als Unterrichts- und Behördensprache (Matthee 2016).

Das bisher ehrgeizigste Projekt ist das eines „Afrikaaner-Zions“, einer im ariden und nur dünn besiedelten privaten (!) ,Afrikaaner-Stadt“ namens Orania am Oranjefluss (Scheen 2013; Drechsler 2015; Bröll 2019). Diese „Stadt“ ist selbst heute, 
gut 30 Jahre nach ihrer Gründung, kaum mehr als ein großes Dorf mit ca. 2000 Einwohnern. Ihre Existenz wurde im Jahr 2000 gerichtlich bestätigt, als das Bemühen der Zentralregierung, Orania einer Nachbargemeinde (Hopetown) einzuverleiben, abgelehnt wurde. So blieb Orania ein Dorf Weißer Afrikaaner; d. h. nicht zuletzt, dass nur Angehörige dieser Bevölkerungsgruppe dort Arbeit finden. Geldenhuys (2021) spricht hier von nicht nur funktionaler, sondern auch territorialer Autonomie. Geplant ist der Ausbau weiterer Siedlungen nach dem Vorbild Oranias über die nördliche Kap-Provinz hinaus. Dazu hat Solidariteit eine größere Zahl von „Ankerdörfern" identifiziert, in denen bereits heute eine große Zahl von Afrikaanern lebt. Die Planungen sind, ihre Zahl so weit als möglich zu erhöhen. Anschließend, so die Hoffnung, werden dort Institutionen afrikaanser Sprache wie Kirchen und Schulen bis hin zu Universitäten entwickelt werden. Die Afrikaanerdörfer, vielleicht irgendwann Afrikaanerstädte, werden nicht zuletzt die sehr vielen immer schon armen oder seit 1994 verarmten Arfikaaner, die im übrigen Südafrika keine Chance haben, aufnehmen. Hier werden sie im Sinne des Accords on Afrikaner Self-Determination vom 23. April 1994 selbstbestimmt leben. Solidariteit ist entschlossen, die Konzentration dieser Bevölkerungsgruppe auf das Intensivste zu fördern. Viele Einzelheiten von alledem sind unbekannt, das schon deshalb, weil sie selbst unter den Afrikaanern strittig sind. Kein Außenstehender wird darüber informiert sein, welche Planungen das Forschungsinstitut von Solidariteit für welche Szenarien vorbereitet hat. Was Solidariteit hier in Zukunft unternimmt, wird nicht zuletzt davon abhängen, wie der gegenwärtige Machtkampf zwischen dem Lager Ramaphosas und dem von Magashule auf Dauer (!) ausgeht (s. Kap. 4). Obsiegt der Letztere, so wird Solidariteit mit Sicherheit auf die auch ihr detailliert bekannten Bahr/Lambsdorffschen Pläne von einem Weiß-Coloured Volksstaat, wahrscheinlich in Teilen des nördlichen/westlichen Kap, zurückkommen. Selbst im Fall eines Sieges Ramaphosas wird das aber auch dann der Fall sein, sollte dessen Regierung ihre Pläne von einer entschädigungslosen Enteignung privaten Eigentums nach simbabwischem Vorbild in die Tat umsetzen.

\section{Die Afrikaaner-Afrika-Initiative als Mediator}

Die sich ständig verschärfende Gefahr der Implosion und ethnische Polarisierung vor Augen hat der frühere Diplomat und Geschäftsmann Chris Opperman zusammen mit Gleichgesinnten, darunter der Philosoph Carel Boshoff jun., vor wenigen Jahren die Afrikaaner-Afrika-Initiative (AAI) gegründet. Bislang vor allem in Zusammenarbeit mit der Thabo Mbeki Foundation (TMF), deren sehr fähigem Sonderbeauftragten Vincent Maphai, und (künftig) Repräsentanten der Nachbarstaaten ist Opperman bemüht, die Selbstzerstörung Südafrikas aufzuhalten. Als Anknüpfungspunkt dient hier die inzwischen historische Konferenz von Dakar im Jahr 1987, zu der van Zyl Slabbert und Mbeki 75 Teilnehmer, darunter den Verfasser dieses Beitrages, in die senegalesische Hauptstadt eingeladen hatten.

Eine erste, von Opperman und Maphai mit großer Sorgfalt vorbereitete Konferenz führte Mbeki und weitere Vertreter seiner TMF sowie die amtierende Ministerin für Landwirtschaft und Landreform, Thoko Didiza, und, unter dem Schirm von 
AAI, Repräsentanten führender Afrikaaner-Organisationen Ende Februar 2021 in Newlands/Kapstadt zusammen. Zu den letzteren gehörte mit Flip Buys und Kallie Kriel die Führungsriege von Solidariteit/AfriForum. Finanziert wurde sie von einer deutschen Stiftung. Zwei Themenkreise beherrschten die Diskussionen (Buys 2021): zum einen die berechtigten Klagen der Afrikaaner, seit Mai 1994 nur noch Bürger zweiter Klasse, bzw. Fremde im eigenen Land zu sein. So forderten sie Teilhabe an der politischen Macht, wo diese über ihre Belange, d. h. vor allem ihre Kultur (Sprache!) entscheidet. Hier forderten sie Selbstbestimmung. Zum anderen boten die Afrikaaner ihre vielfältige Mitwirkung beim Wiederaufbau des Staates an. Wird dieses Angebot angenommen, dann wird, um hier nur ein Beispiel herauszugreifen, das von AfriForum entwickelte „Community- and technology based security system“ Bestandteil des staatlichen Sicherheitsapparates sein.

Nicht anders als in Dakar taten sich die Vertreter des ANC auch jetzt in Kapstadt mit Kritik an seiner Politik schwer. Diese auch heute weltweit hofierte Bewegung ist es schlichtweg nicht gewohnt, kritisch gesehen zu werden. Auch werden in der Zeit des Exils in der kommunistischen Welt entstandene Vorbehalte gegen Minderheitenschutz weiterhin vorhanden sein. Zudem fehlt es dem ANC nach 350 Jahren eines rassistischen Regimes mit guten Gründen am Vertrauen dahingehend, dass die Unterdrücker von einst binnen eines Vierteljahrhunderts zu loyalen Bürgern der neuen Kap-Republik geworden sind. Dementsprechend wurden, so wie vor Jahr und Tag in Dakar, Problemkreise wie „sacrificial partition“"12, d. h. die Schaffung eines Afrikaaner-Israel, mit keinem Wort angesprochen. ${ }^{13}$

Zweierlei sollte aber für Südafrika bescheidene Hoffnung geben: Die Vertreter des Schwarzen Südafrikas waren in Kapstadt nicht nur bereit, die sehr deutlich vorgetragenen Klagen der Afrikaaner anzuhören, sondern sie auch in die gemeinsame Abschlusserklärung (,Joint Declaration“) aufzunehmen (Politicsweb 2021). Außerdem sind beide Seiten fest entschlossen, den für die Zukunft vielleicht von Solidariteit zu übernehmenden Meinungsaustausch mit Hilfe der bereits jetzt vorbildlich engagierten deutschen Stiftung fortzusetzen. Wenngleich es durchaus auch kritische Stimmen zu der Rolle von Solidariteit gibt (Zyl-Hermann 2018): Ohne den beschriebenen Austausch ist die Wahrscheinlichkeit hoch, dass die Afrikaaner eine Teilung des Landes anstreben werden. Dies birgt die Gefahr eines südafrikanischen Bürgerkrieges, der viel verbrannte Erde zurücklassen würde. Vor einem solchen Szenario hat der Maler und in Apartheidzeiten langjährige politische Häftling Breyten Breytenbach bereits am 5. Juni 1991 im Gespräch mit der französischen Tageszeitung Le Monde gewarnt: „L'Afrique du Sud va bientôt explorer les variantes infinies de la barbarie“ (Le Monde 1991, „Südafrika wird in absehbarer Zukunft die unendlichen Varianten der Barbarei entdecken“, eigene Übersetzung).

Danksagung Der Verfasser widmet seinen Beitrag seinem langjährigen Freund Professor Dr. Deon Geldenhuys, ehemals Randse Afrikaanse Universiteit, Johannesburg, Suid Afrika.

\footnotetext{
12 Dieser Ausdruck wurde von van Zyl Slabbert geprägt und wiederholt genutzt.

13 Diesen Punkt anzusprechen oblag in Dakar einem der ausländischem Mitglieder der Slabbert-Delegation (Ropp 1989a und 1989b).
} 


\section{Literatur}

Adam, K. (1997). The politics of redress: South African style affirmative action. The Journal of Modern African Studies, 35(2), 231-249.

Alexander, N. (2007). Affirmative action and the perpetuation of racial identities in post-apartheid South Africa. Transformation: Critical Perspectives on Southern Africa, 63, 92-108.

von Beyme, K. (2001). Russland zwischen Anarchie und Autokratie. Wiesbaden: VS.

Bond, P. (2014). South Africa's resource curses and growing social resistance. Monthly Review, 65(11), $1-21$.

Bröll, C. (2008). Fachkräfte flüchten aus Südafrika. https://www.faz.net/aktuell/karriere-hochschule/ buero-co/zur-auswanderung-getrieben-fachkraefte-fluechten-aus-suedafrika-1548323-p2.html. Zugegriffen: 30. Juli 2021.

Bröll, C. (2019). Ein weißes Feld. Frankfurter Allgemeine Magazin, Bd. 20. Juni (S. 35-39).

Buys, F. (2021). A perspective on the Afrikaner talks with the Mbeki Foundation. www.politicsweb.co.za/ opinion/a-perspective-on-the-afrikaner-talks-with-the-mbek. Zugegriffen: 1. Juli 2021.

Cruywagen, D. (2014). Brothers in war and peace. Constand and Abraham Viljoen and the birth of the New South Africa. Cape Town: Random House Struik.

Cuddihy, M. (2016). White South Afrikans complain affirmative action policy is causing them to face discrimination. https://www.abc.net.au/news/2016-08-01/white-south-africans-complain-of-a-reverseapartheid/7676764. Zugegriffen: 30. Juli 2021.

Drechsler, W. (2015). Mit der Weisheit am Ende. Der Tagesspiegel, Bd. 30. Mai (S. 27).

Erasmus, Z. (2015). The nation, its population and their re-calibration: South African affirmative action in a neoliberal age. Cultural Dynamics, 100-115.

Fukuyama, F. (1991). The next South Africa. The National Interest, 18, 13-28.

Geldenhuys, D. (2021). Autonomy initiatives of the Afrikaner community in South Africa. In T. H. Malloy \& L. Salat (Hrsg.), Non-territorial autonomy and decentralisation. Ethno cultural diversity governance (S. 96-105). London: Routledge.

Geschke, G., \& Mack, G. (1977). „Ohne Verhandlungslösung ist die Gefahr des dritten Weltkriegs ständig gegenwärtig“. Interview mit Egon Bahr. Deutsches Allgemeines Sonntagsblatt, 28, 8.

Government Communication and Information System (2001). South Africa yearbook 2001/02 (8. Aufl.). Pretoria: GCIS.

Government of South Africa (2021). South Africa's people. https://www.gov.za/about-sa/south-africaspeople. Zugegriffen: 5. Juli 2021.

Grimm, D. (2020). Das Grundgesetz - Ein Exportartikel. In A. Fahrmeier (Hrsg.), Deutschland. Globalgeschichte einer Nation (S. 683-687). München: C.H. Beck.

Hunter, M. (2019). Race for education: Gender, White tone, and schooling in South Africa (The International African Library). Cambridge: Cambridge University Press. https://doi.org/10.1017/ 9781108635189.

Grundlingh, A. (2021). Slabbert. Man on a mission. A biography. Johannesburg: Jonathan Ball.

Johnson, R. W. (2021a). Thinking about state failure (III). https://www.politicsweb.co.za/opinion/thinkingabout-state-failure-iii-2. Zugegriffen: 1. Juli 2021.

Johnson, R. W. (2021b). An almost indescribable mess. https://www.politicsweb.co.za/opinion/an-almostindescribable-mess. Zugegriffen: 1. Juli 2021.

Kappel, R. (2019). Südafrika schlittert noch weiter in die soziale und wirtschaftliche Krise. In Deutsche Gesellschaft für die Vereinten Nationen (Hrsg.), Blickpunkt Südafrika. Blaue Reihe, (Bd. 117, S. 51-56). Berlin: Deutsche Gesellschaft für die Vereinten Nationen.

Graf Lambsdorff, O. (1986). Teilung Südafrikas als Ausweg. Quick, Bd. 31. Juli (S. 32).

Le Monde (1991). Afrique du Sud/L'écrivain Breyten Breytenbach dénonce ,culture stalinienne“ de l'ANC. https://www.lemonde.fr/archives/article/1991/06/05/afrique-du-sud-1-ecrivain-breytenbreytenbach-denonce-la-culture-stalinienne-de-1-anc_3999124_1819218.html. Zugegriffen: 5. Juli 2021.

Makoba, J., \& Ntebeng, M. (2002). Affirmative action policy and the search for racial equality identities in post-apartheid South Africa. India Quarterly, 58(1), 165-176.

March, L. (2021). Die Ursachen für Chaos und Gewalt. https://www.deutschlandfunk.de/hintergrund.723. de.html?drbm:date=2021-07-23. Zugegriffen: 30. Juli 2021.

Matthee, H. (2016). Taalbelang is stoffelijk belang! - In Südafrika ist die Sprache Afrikaans durch Einheitsenglisch bedroht. Deutsche Sprachwelt, 3, 7.

Ocampo, M.L. (2004). A brief history of educational inequality from Apartheid to the present. https://web. stanford.edu/ jbaugh/saw/Lizet_Education_Inequity.html. Zugegriffen: 30. Juli 2021. 
Pabst, M. (2019). Südafrika und die Vereinten Nationen - Ein Rückblick auf die Jahre 1945 bis 1994. In Deutsche Gesellschaft für die Vereinten Nationen (Hrsg.), Blickpunkt Südafrika. Blaue Reihe, (Bd. 11, S. 5-36). Berlin: Deutsche Gesellschaft für die Vereinten Nationen.

Pabst, M., \& Freiherr von der Ropp, K. (2011). Eine kritische Würdigung der deutschen Südafrika Politik. Zeitschrift für Außen- und Sicherheitspolitik, 4(4), 31-42.

du Plessis, L. M. (2008). German Verfassungsrecht under the Southern Cross. Observations on South African-German interaction in recent history with particular reference to constitution-making in South Africa. In F. Hufen (Hrsg.), Verfassungen zwischen Recht und Politik. Festschrift zum 70. Geburtstag für Hans-Peter Schneider (S. 524-536). Baden-Baden: Nomos.

Politicsweb (2021). Declaration of the Afrikaner - Africa Conference. https://www.politicsweb.co.za/ documents/declaration-of-the-afrikaner--africa-conference. Zugegriffen: 30. Juli 2021.

Poplak, R. (2021). Ace Magashule, South Africa's next president? https://www.dailymaverick.co.za/ article/2021-02-13-ace-magashule-south-africas-next-president/. Zugegriffen: 8. Juli 2021.

Ramphele, M. (2012a). Tell my people that I love them and that they must continue the struggle. Solomon Mahlangu Memorial Lecture. Johannesburg: Centre for Education Policy Development.

Ramphele, M. (2012b). Conversations with my sons and daughters. Johannesburg: Penguin.

Ratuva, S. (2013). Politics of preferential development: trans-global study of affirmative action and ethnic conflict in Fiji, Malaysia and South Africa. Canberra: ANU Press.

Robertson, M. (1991). Sir Robin - his excellent excellency. Sunday Times Johannesburg, Bd. 20. Apr. (S. 14).

Roche, D. (1998). South Africa's capital crises. The Wallstreet Journal Europe, Bd. 9. Sept. (S. 10).

Freiherr von der Ropp, K. (1989a). Two years after Dakar, the dialogue continues. Democracy in Action, 7, 14-15.

Freiherr von der Ropp, K. (1989b). Afrikaner - Israel kan'n tweede Lebanon verhoed. Vrye Weekblad, Bd. 27. Okt. (S. 5).

Freiherr von der Ropp, K. (2013). Das neue Südafrika. Vom Umbruch zum Zusammenbruch? Eine deutsche Sicht. Zeitschrift für Außen- und Sicherheitspolitik, 6(3), 357-368.

Freiherr von der Ropp, K. (2017). Offener Brief an einen verstorbenen Freund: Hat Orania eine Chance? http://www.africanquestions.org/articles/offener-brief-an-einen-verstorbenen-freund-hat-oraniaeine-chance-mit-einem-vorwort-von-constand-viljoen-01-march-2017-75. Zugegriffen: 28. Juli 2021.

Freiherr von der Ropp, K. (2018). Das Post-Apartheid-Südafrika. Zu dem Treffen von Afrikanern und Afrikaanern in Dakar. In U. van der Heyden (Hrsg.), Der Dakar-Prozess (S. 125-137). Kiel: Solivagus.

Freiherr von der Ropp, K. (2019). Der Niedergang des demokratischen Südafrika. Ein deutscher Beitrag. In Deutsche Gesellschaft für die Vereinten Nationen (Hrsg.), Blickpunkt Südafrika. Blaue Reihe, (Bd. 11, S. 59-69). Berlin: Deutsche Gesellschaft für die Vereinten Nationen.

Runge, K. K. (2015). Zuwanderung. https://www.bpb.de/gesellschaft/migration/laenderprofile/200200/ zuwanderung?p=0. Zugegriffen: 30. Juli 2021.

Scheen, T. (2013). In der Wagenburg. Frankfurter Allgemeine, Bd. 1. Juni (S. 3).

von Soest, C. (2020). Südafrika. http://www.bpb.de/internationales/weltweit/innerstaatliche-konflikte/ 54809/suedafrika. Zugegriffen: 30. Juli 2021.

Statistics South Africa (2021). Quarterly Labour Force Survey. Quarter 1: 2021. Pretoria: Republic of South Africa.

Steininger, R. (2009). Faszinierende Lektüre. Akten zur Außenpolitik der Bundesrepublik Deutschland. Frankfurter Allgemeine, Bd. 27. Aug. (S. 7).

Swilling, M., Bhorat, H., Buthelezi, M., Chipkin, I., Duma, S., Mondi, L., Peter, C., \& Qobo, M. (2017). Betrayal of the promise. How South Africa is being stolen. State Capacity Research Project. http:// 47zhcvti0ul2ftip9rxo9fj9.wpengine.netdna-cdn.com/wp-content/uploads/2017/05/Betrayal-of-thePromise-25052017.pdf. Zugegriffen: 1. Juli 2021.

Tagesschau (2021). Gewalt in Südafrika weitet sich aus. https://www.tagesschau.de/ausland/afrika/ suedafrika-proteste-pluenderungen-103.html. Zugegriffen: 19. Juli 2021.

The Nelson Mandela Foundation (1994). Accord on Afrikaner self-determination 23. April 1994. https:// omalley.nelsonmandela.org/omalley/index.php/site/q/031v02039/04lv02103/051v02120/061v02123. htm. Zugegriffen: 5. Juli 2021.

The World Bank (2021a). GDP growth (annual \%) - South Africa. https://data.worldbank.org/indicator/ NY.GDP.MKTP.KD.ZG?contextual=default\&end=2020\&locations=ZA\&most_recent_year_desc=fa lse\&start=1961\&view=chart. Zugegriffen: 5. Juli 2021.

The World Bank (2021b). Population total - South Africa. https://data.worldbank.org/indicator/SP.POP. TOTL?locations=ZA. Zugegriffen: 9. Juli 2021. 
Vergau, H.-J. (2002). Genscher und das südliche Afrika. In H.-D. Lucas (Hrsg.), Genscher Deutschland und Europa (S. 223-239). Baden-Baden: Nomos.

Waldmeir, P. (1991). Farewell to the host with the most in South Africa. Financial Times, Bd. 1. Juni (S. 3).

Waldmeir, P., \& Holman, M. (1994). Spirit of reconciliation sweeps aside letter of vote. The election was deeply flawed, but nearly everyone likes the outcome. Financial Times, Bd. 7./8. Mai (S. 3).

Weber, B. (2011). Carel Boshoff, founder of White redoubt in South Africa, dies at 83. The New York Times. https://www.nytimes.com/2011/03/20/world/africa/20boshoff.html. Zugegriffen: 30. Juli 2021.

Webster, D. (2019). An indictment of South Africa: Whites-only town Orania is booming. https:// www.theguardian.com/cities/2019/oct/24/an-indictment-of-south-africa-whites-only-town-oraniais-booming. Zugegriffen: 30. Juli 2021.

Winning, A., \& Bavier, J. (2019). Ramaphosa is last hope for SA, says Chinese ambassador. https:// www.businesslive.co.za/bd/economy/2019-07-29-ramaphosa-is-last-hope-for-sa-says-chineseambassado. Zugegriffen: 1. Juli 2021.

World Economic Forum (2017). The Global competitiveness report 2017-2018. Genf: World Economic Forum.

Zollmann, J. (2021). Negotiated partition of South Africa - an idea and its history (1920s-1980s). South African Historical Journal. https://doi.org/10.1080/02582473.2021.1909119.

van Zyl-Hermann, D. (2018). Make Afrikaners great again! National populism, democracy and the new white minority politics in post-apartheid South Africa. Ethnic and Racial Studies, 41(15), 2673-2692. https://doi.org/10.1080/01419870.2017.1413202. 